\title{
Micromechanical modeling of the interactions between the microstructure and the dissipative deformation mechanisms in steels under cyclic loading
}

\author{
Charles Mareau ${ }^{\mathrm{a}}$, Véronique Favier ${ }^{\mathrm{b}}$, Bastien Weber $^{\mathrm{c}}$, André Galtier ${ }^{\mathrm{d}}$, \\ Marcel Berveiller ${ }^{\mathrm{a}}$ \\ a Arts et Métiers PARISTECH, Laboratoire de Physique et Mécanique des Matériaux UMR CNRS \\ 7554, 4 rue Augustin Fresnel 57078 METZ CEDEX 3, France \\ b Arts et Métiers PARISTECH, Laboratoire Procédés et Ingénierie en Mécanique et Matériaux, UMR \\ CNRS 8006, 151 Boulevard de l'Hôpital 75013 PARIS, France \\ ${ }^{\mathrm{c}}$ ArcelorMittal Maizières Research and Development Voie Romaine 57280 MAIZIERES-LES-METZ \\ d CETIM 52 Avenue Félix-Louat 60304 SENLIS CEDEX, France
}

\begin{abstract}
A micromechanical model is proposed to describe the interactions between the microstructure and the dissipative deformation mechanisms in ferritic steels under cyclic loading. The model aims at optimizing the microstructure of steels since the dissipative mechanisms can be responsible for the initiation of microcracks. Therefore, a better understanding of the influence of the microstructure could lead to an improvement of fatigue properties. The dissipative mechanisms are assumed to be either anelastic (dislocation oscillations) or inelastic (plastic slip) and are described at the scale of the slip system using the framework of crystal plasticity. The macroscopic behavior is then deduced with a homogenization scheme. The model is validated by comparing the simulations with experimental results and is finally used to predict the impact of different microstructure parameters on the heat dissipation.
\end{abstract}

Key words: fatigue, microplasticity, polycrystalline material, dislocations

\section{Introduction}

Fatigue design requires the determination of the conventional fatigue limit using commonly very long and costly testing. Methods based on self-heating measurements were

Email address: charles.mareau@ensam.eu (Charles Mareau).

Preprint submitted to Elsevier

6 December 2011 
developed for the rapid determination of the fatigue limit (Gomaa, 1980; Luong, 1998; La Rosa and Risitano, 2000; Poncelet et al., 2010; Munier et al., 2010). They are based on the experimental observation that a material undergoing a fatigue test is subjected to an increase of the surface temperature. This increase is related to progressive microstructure changes up to the specimen rupture. Although realistic estimations of the fatigue limit were obtained with this fast determination method, it can also lead to questionable results since the link between fatigue properties and self-heating remains empirical (Morabito et al., 2002; Chrysochoos et al., 2008). Some authors rather use temperature measurements to predict the influence of microstructure on fatigue properties (Galtier et al., 2002). Indeed, the initiation of microcracks is caused by mechanisms occurring at a microscopic scale that are irreversible in the sense of thermodynamics. So, in high cycle fatigue (i.e. when the microcracks initiation represents the main part of the lifetime), if the microstructure is modified in order to reduce the temperature increase (so the intensity of the irreversible mechanisms), the fatigue properties are very likely to be improved. However, experimental investigations of the interactions between the microstructure and the dissipative mechanisms display limitations. First, although the recent development of infrared thermography enable to identify two dimension dissipation fields, the spatial resolution is not high enough to estimate the value of the dissipation field at a microscopic scale (i.e. inside grains) (Boulanger et al., 2004; Berthel et al., 2007; Chrysochoos et al., 2008). Moreover, because of metallurgical restrictions, microstructural parameters are often interrelated which makes difficult the analysis of one single parameter on the dissipation. For example, changing the mean grain size of metallic alloys by heat treatments also changes the grain size distribution.

The objectives of the paper are to model and analyze the influence of some microstructural parameters that can be controlled by processing such as the volume fraction of precipitates, the grain size distribution and the initial texture on the intrinsic dissipation produced under cyclic loading in the case of bcc (body-centered cubic) ferritic steels. Typical stress amplitudes, used for rapid estimation of the fatigue limit by self-heating measurements, range from $0.15 \sigma_{y}$ to $0.6 \sigma_{y}$ where $\sigma_{y}$ is the conventional yield stress. So they are around the microyield stress. Consequently, the (visco)plastic strain is very small and inferior to $10^{-4}$. The number of cycles is also quite low and is equal to 40000 in the present case. This number of cycles ensures the thermal steady state. The objectives of the work required correlating the dissipative response to the deformation mechanisms and the microstructure described by statistical representations. To this end, we formulated a rate-dependent constitutive equation at the grain scale accounting for three mechanisms: elasticity, anelasticity and inelasticity using the crystal plasticity framework. This constitutive equation was incorporated into a polycrystalline model based on the self-consistent-type approximation developed by (Mareau et al., 2009a) describing elastic-anelastic-inelastic interactions between grains. Self-consistent models already showed with success their ability to associate the overall response to the polycrystalline microstructure and deformation mechanisms (Paquin et al., 2001; Berbenni et al., 2007). The polycrystalline model is used here to clarify the role of microstructural parameters separately on the overall response. These microstructural parameters are described by their mean value or distribution law. Section 2 aims at identifying and modeling the main dissipative mechanisms related to the deformation of bcc ferritic steels at the grain scale and around the microyield stress. Section 3 deals with the scale transition scheme suitable for an elastic-anelastic-inelastic behavior. Section 4 is dedicated to the prediction 
of the dissipative response of ferritic steels having various microstructures.

\section{Description of the dissipative mechanisms}

\subsection{Identification of the dissipative mechanisms}

Figure 1 shows the temperature increase versus time for a low-alloyed ferritic steel specimen submitted to a cyclic loading having increasing stress amplitudes from $50 \mathrm{MPa}$ up to $290 \mathrm{MPa}$. Although it is plotted for a low-alloyed ferritic steel, it is typical for any ferritic steel. For a given stress amplitude $\Sigma_{a}$, the surface temperature rapidly increases at the beginning of the test and then it tends to a stationary value (associated with the thermal steady state). An additional rapid temperature increase up to a new stationary value is observed when the stress amplitude is increased. When the specimen is unloaded, the temperature decreases to a value close to zero associated with a new thermal equilibrium. When a periodic uniaxial stress is applied to a specimen, the thermodynamical framework proposed by Louche and Chrysochoos (2001) and Boulanger (2004) enables to estimate the average (over the volume and over one loading cycle) dissipation per cycle $\tilde{d}_{1}$ from the stationary mean temperature increase per cycle $\tilde{\theta}$ according to the following relation:

$$
\frac{\tilde{d}_{1}}{m_{v} C_{p}}=\frac{\tilde{\theta}}{\tau}
$$

The dissipation is generally divided by the mass density $m_{v}$ and the specific heat $C_{p}$ so it is expressed in $K / s$ rather than in $\mathrm{W} / \mathrm{m}^{3} . \tau$ is a time constant that characterizes the thermal exchanges between the sample and its environment and is easily determined during experiments. In the above equation, the average temperature increase per cycle is not related to the thermoelastic source since the thermoelastic energy vanishes at the end of each complete loading cycle (Boulanger et al., 2004). Moreover, the present form of the heat diffusion equation is valid as long as heat sources are considered to be uniformly distributed over the gauge volume (Boulanger et al., 2004). In our case, this assumption is valid since the material grain size is much smaller than the gauge size.

In the present paper, experimental results were obtained by measuring the temperature with a type $\mathrm{T}$ thermocouple placed at the specimen surface. The thermocouple measures an average temperature over a $1 \mathrm{~mm}^{2}$ area at the specimen centre. The temperature is assumed to be quasi-uniform on this area because of the high thermal conductivity coefficient of steel. Since specimens are thin $(2 \mathrm{~mm})$, we assumed that the temperature along the specimen thickness is uniform and equal to the surface temperature.

Following Boulanger et al. (2004) and Berthel et al. (2007), we estimated $\tilde{d}_{1} /\left(m_{v} C_{p}\right)$ in the stationary regime for various stress amplitudes and for constant frequency $f_{s}=$ $1 / T_{s}=30 \mathrm{~Hz}$ and stress ratio $R_{s}$ (defined as the minimal stress divided by the maximal stress of the periodic loading). For a given stress amplitude, we experimentally observed that 5000 cycles are sufficient to reach the thermal steady state. Note that the thermal and mechanical properties of the material are assumed constant throughout the tests since the temperature rise is lower than $10 \mathrm{~K}$. Moreover, material degradation due to fatigue damage is not expected to play a significant role here since the number of applied cycles is much lower than the number of cycles causing fracture at those stresses. This assumption is in agreement with the experimental observations of Chrysochoos et al. 


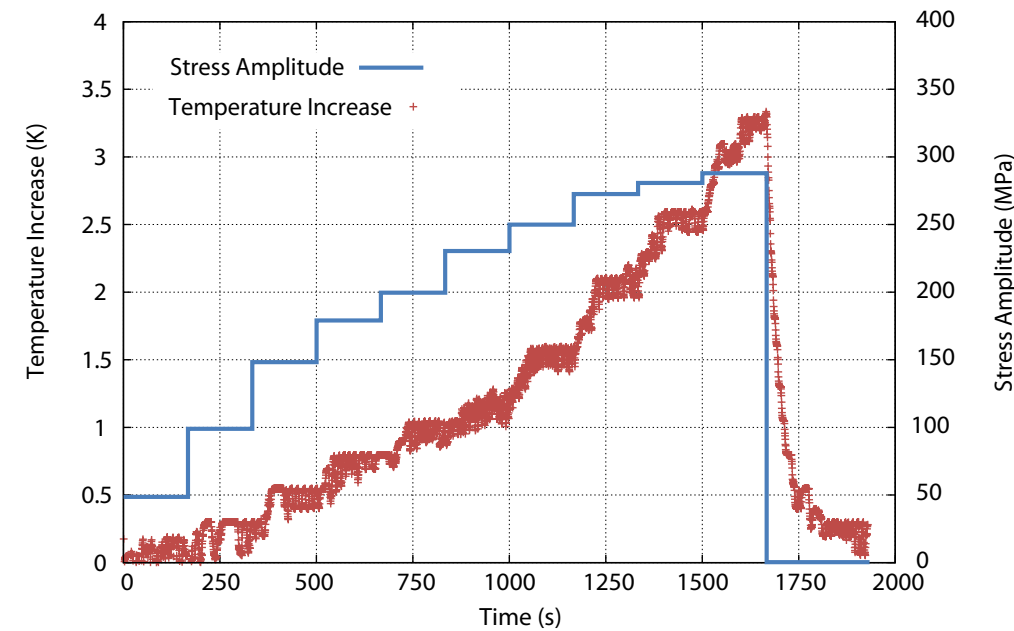

Fig. 1. Evolution of the temperature and stress amplitude as a function of time in the case of a low alloyed ferritic steel specimen submitted to a cyclic stress whose amplitude varies from 50 to $290 \mathrm{MPa}$ with a loading frequency of $30 \mathrm{~Hz}$ and a stress ratio of -1

(2008) who did not detect an influence of fatigue damage on the elastic parameters up to 124000 cycles in the case of a dual-phase (ferrite and martensite) steel.

Plotting the average dissipation per cycle versus the stress amplitude results in figure 2, labeled as self-heating curves. Mareau et al. (2009b) showed that the dissipative mechanisms are different depending on the stress amplitude. For low stress amplitudes, the dissipation is independent on the loading ratio and is due to an anelastic mechanism (i.e. the strain is irreversible but recoverable from a mechanical point of view). For high stress amplitudes, the dissipative mechanism is inelastic (i.e. the strain is irreversible and unrecoverable) since the dissipation is strongly influenced by the loading ratio. The transition from an anelastic to an inelastic dissipative mechanism is responsible for the strong dissipation increase that is observed from a critical stress amplitude $\Sigma_{c}$ as shown on figure 2 for a HSLA steel (i.e. a low-alloyed ferritic steel). The existence of two different mechanisms was shown by Maquin and Pierron (2009) and later by Connesson et al. (2010) using other arguments. They analyzed the dissipated energy during the first 
cycles for various stress levels, stress ratios and metallic alloys. In particular, Maquin and Pierron (2009) compared the dissipated energy for increasing stress levels using either a new virgin specimen for each test or the same specimen. They found that for lower stress levels, the dissipated energy for both kinds of specimen was the same whereas it was different for higher stress levels. These results show that no microstructure change occurs during the low stress level experiments so that the material undergoes a anelastic behaviour. For higher stress levels, microplasticity took place leading to different microstructures for the two kinds of specimens which had two different mechanical histories (Maquin and Pierron, 2009). Connesson et al. (2010) measured a significant phase shift between stress and strain in a ferritic steel under cyclic loading and conclude the presence of visco-elastic mechanisms. In sections 2.1.1 and 2.1.2, we model the anelastic and inelastic mechanisms at the grain scale using the crystal plasticity framework.

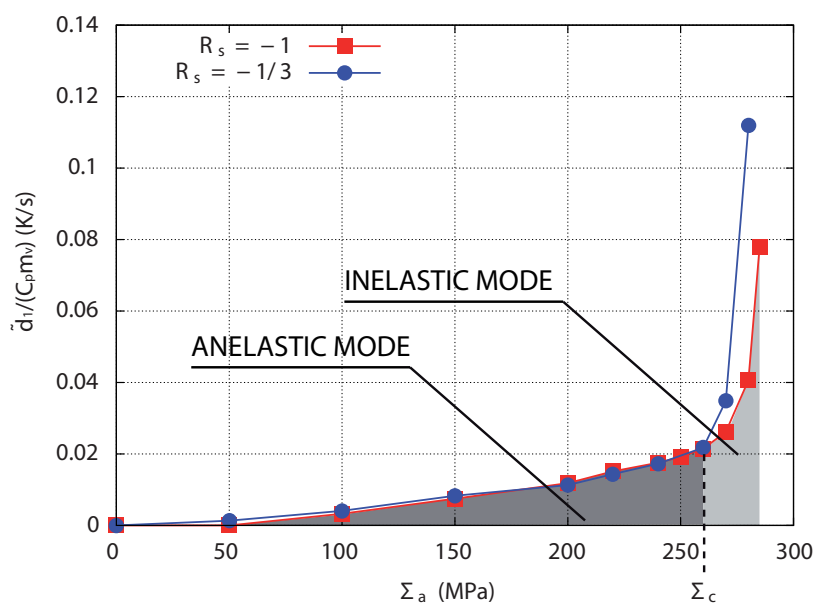

Fig. 2. Experimental self-heating curves, namely average dissipation per cycle versus stress amplitude, obtained for a ferritic steel hardened with precipitates (HSLA) for different loading ratios.

\subsubsection{Anelastic mechanism}

The anelastic mechanism is difficult to identify since there is a large number of possible anelastic dissipative mechanisms. Indeed, the dissipation can either be due to the migration of atoms like in a Snoek/Köster (Snoek, 1941) or Zener type mechanism or to a dislocation relaxation phenomena as proposed by Koehler (1952) (i.e. the motion of dislocations is analogous to the motion of a damped vibrating string). Since, for bcc metals, Seeger (2004) mentioned the existence of a relaxation peak at low frequencies (i.e. under the $\mathrm{kHz}$ range) and at room temperature that could be attributed to dislocations, the anelastic mechanism being responsible for the dissipation at low stress amplitudes is likely to be due to a dislocation relaxation phenomena.

To confirm the origin of the anelastic mechanism, self-heating curves were made for a ferrite-bainite steel in two cases: after a $10 \%$ tensile prestrain and without prestrain. Results are displayed on figure 3 . The prestrain induces a strong increase of the dissipation in both regimes (anelastic and inelastic) that is obviously due to the multiplication of dislocations. In other words, there is a strong coupling between the dislocation density 
and the anelastic mechanism. As a result, one possible anelastic mechanism is the oscillation of pinned dislocations. The recoverability of such a mechanism is due to line tension that is responsible for a backstress bringing dislocation lines back in their equilibrium position. However, the anelastic displacements of the pinned dislocations are irreversible because of lattice friction. It is worth noticing that the anelastic displacements are related to the bowing of edge dislocations whereas inelastic glide requires the motion of screw dislocations (see section 2.1.2) (Brown and Ekvall, 1962; Solomon and McMahon, 1966; Sommer et al., 1998; Naamane et al., 2010). In the following modeling, we consider this mechanism as the only anelastic mechanism.

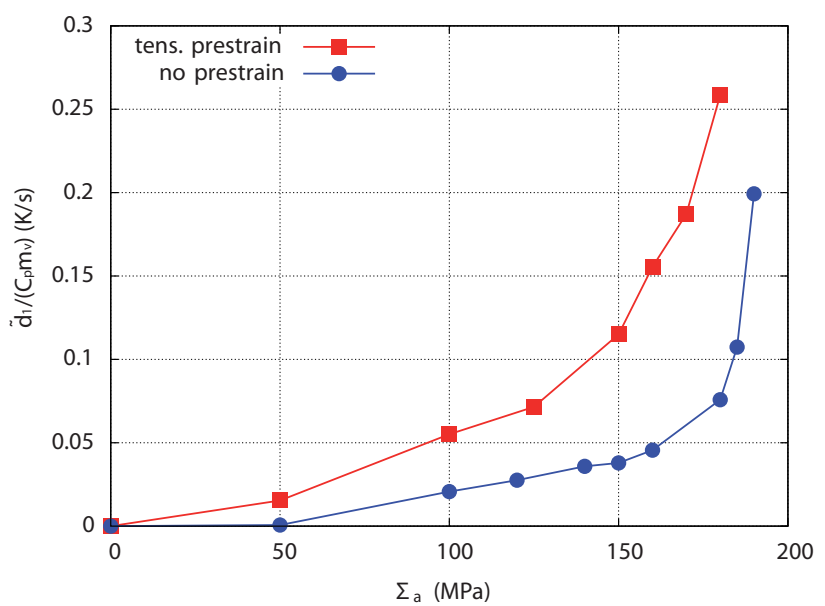

Fig. 3. Experimental self-heating curves, namely average dissipation per cycle versus stress amplitude, obtained for a ferrite-bainite steel without prestrain (no prestrain.) and after a tensile prestrain of $10 \%$ (tens. prestrain).

The slip rate of a slip system induced by the anelastic mechanism is deduced from the forces equilibrium condition applied to a bowed dislocation line pinned between two obstacles (as described on figure 4). Considering such a single dislocation line with an infinitesimal length $d r$, the different forces are:

- The Peach-Kohler force $\vec{F}_{\tau}$ induced by the effective stress field (supposed to be uniform):

$$
\vec{F}_{\tau}=\tau_{\text {eff }}^{g} b^{g} d r \vec{j}
$$

where $\tau_{\text {eff }}^{g}$ is the effective resolved shear stress, $b^{g}$ the magnitude of the Burgers vector and $\vec{j}$ is the unit vector defining the slip direction in the slip plane. The calculation of the resolved shear stress takes into account the presence of kinematic stress inside a grain and is given in equation (22).

- The friction force $\vec{F}_{f}$ opposing the motion of the dislocation and assumed to be proportional to the displacement rate:

$$
\vec{F}_{f}=-f \dot{u} d r \vec{j}
$$

where $f$ is the friction coefficient associated with the lattice friction for edge dislocations. 
- The line tension $\overrightarrow{d \Gamma}$ responsible for the recoverability of the anelastic strain:

$$
\overrightarrow{d \Gamma}=\mu b^{g^{2}} \frac{\overrightarrow{\partial u}}{\partial r}
$$

where $\mu$ is the shear modulus.

(a)

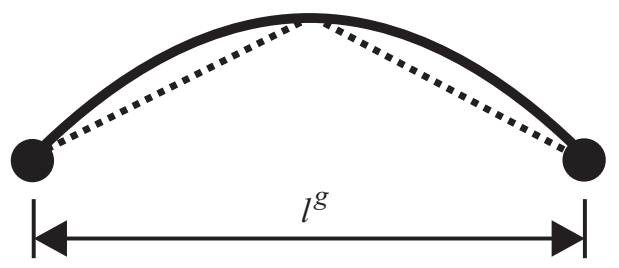

(b)

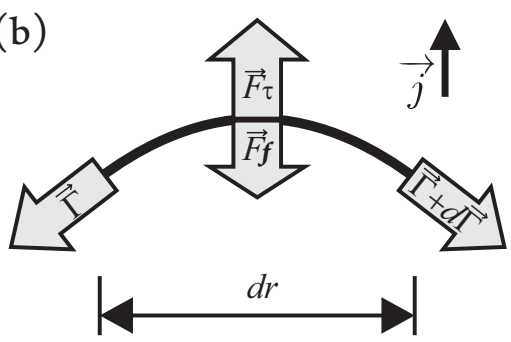

Fig. 4. (a) Schematic drawing of a dislocation line pinned between two obstacles. The dashed line represents the simplified geometry used to calculate the slip rate. (b) Forces applied to an infinitesimal dislocation segment.

The loading frequencies being lower than $1 \mathrm{kHz}$, the inertial effects are neglected (Granato and Lücke, 1956) so the displacement field of the dislocation is ruled by the following differential equation:

$$
\tau_{e f f}^{g} b^{g}-f \dot{u}+\mu b^{g^{2}} \frac{\partial^{2} u}{\partial r^{2}}=0 .
$$

Simplifying the geometry of the dislocation as shown in figure 4 and integrating the above equation over the length $l^{g}$ of the dislocation line leads to the relation:

$$
\dot{\bar{u}}=\frac{1}{f}\left(\tau_{\text {eff }}^{g} b^{g}-8 \mu b^{g^{2}} \frac{\bar{u}}{l^{g^{2}}}\right)
$$

where $\bar{u}$ (respectively $\dot{\bar{u}}$ ) is the average of the displacement (respectively displacement rate) over the dislocation length. Let us now consider a population of mobile dislocations with density $\rho_{m}^{g}$ on a slip system $g$ and assume that the single dislocation line described above is representative of this population. Following Orowan's relation, the anelastic slip $\gamma_{a n}^{g}$ and slip rate $\dot{\gamma}_{a n}^{g}$ have the following expressions if the mobile dislocations density is assumed to be constant $\left(\dot{\rho}_{m}^{g}=0\right)$ when the strain is anelastic:

$$
\begin{aligned}
& \gamma_{a n}^{g}=\rho_{m}^{g} b^{g} \bar{u}, \\
& \dot{\gamma}_{a n}^{g}=\rho_{m}^{g} b^{g} \dot{\bar{u}} .
\end{aligned}
$$

Finally, the anelastic slip law is obtained by combining the equations 6,7 and 8 :

$$
\dot{\gamma}_{a n}^{g}=\frac{1}{f}\left(\rho_{m}^{g} b^{g^{2}} \tau_{e f f}^{g}-8 \mu b^{g^{2}} \frac{\gamma_{a n}^{g}}{l^{2}}\right) .
$$

In the above equation, the last term represents a backstress that increases with an increasing anelastic deformation and/or with a decreasing mean distance between obstacles. The mean distance between obstacles is determined using the relation proposed by Friedel (1964):

$$
l^{g}=\left(\frac{\mu b^{g}}{\rho_{\text {obst }}^{g} \tau_{\text {eff }}^{g}}\right)^{\frac{1}{3}} .
$$


where $\rho_{\text {obst }}^{g}$ is the total density of obstacles on the $g^{t h}$ slip system. In the present study, two types of obstacles are considered: forest dislocations and precipitates so the total density of obstacles is:

$$
\rho_{\text {obst }}^{g}=\rho^{g}+\frac{3 f_{v}}{2 \pi} \frac{1}{r_{p}^{2}}
$$

where $\rho^{g}$ is the dislocation density on the $g^{t h}$ slip system, $r_{p}$ the precipitate radius and $f_{v}$ the volume fraction of precipitates (Deschamps and Brechet, 1998).

\subsubsection{Inelastic mechanism}

As stated by Galtier et al. (2002), a strong dissipation increase is measured when stress amplitudes are high enough. This increase coincides with the appearance of persistent slip bands so the plastic slip of dislocations is clearly identified as the inelastic dissipative mechanism. Indeed, in bcc iron (which is the main constituent of the studied steels), plastic slip is controlled by screw dislocations whose behavior is temperature and rate dependent (Campbell and Ferguson, 1970; Louchet et al., 1979; Tang et al., 1998) because of the Peierls-Nabarro obstacles (Peïerls, 1940; Nabarro, 1947). Johnston and Gilman (1959) showed that the dislocation velocity obeys an Arrhenius-type law from which Berbenni et al. (2004) proposed the following inelastic slip law:

$$
\dot{\gamma}_{i n}^{g}=\dot{\gamma}_{0}\left(\frac{\tau_{\text {eff }}^{g}}{\mu}\right)^{2} \exp \left(\frac{-\Delta G_{0}}{k_{b} T}\left(1-\left(\frac{\left\langle\left|\tau_{\text {eff }}^{g}\right|-\tau_{c}^{g}\right\rangle}{\hat{\tau}}\right)^{p}\right)^{q}\right) \operatorname{sign}\left(\tau_{\text {eff }}^{g}\right)
$$

where $\langle$.$\rangle denotes the McCaulay brackets, \dot{\gamma}_{0}, p$ and $q$ are materials parameters (with $0<p \leq 1$ and $1 \leq q \leq 2), \Delta G_{0}$ is the activation energy in a stress-free configuration, $T$ is the temperature, $k_{b}$ is the Boltzmann constant, $\hat{\tau}$ is the shear stress necessary to overcome Peierls obstacles at $T=0 \mathrm{~K}$ and $\tau_{c}^{g}$ is the critical shear stress from which the viscoplastic slip is possible.

\subsubsection{Criterion for anelastic-inelastic transition}

Although the dislocations of a given slip system have different characteristics (length, orientation...) so different behaviors (anelastic or inelastic) (Mollica et al., 2001), it is assumed for simplicity that the behavior is the same for all the dislocations of a same slip system. The behavior of dislocations is conditioned by a critical shear stress criterion $\tau_{c}^{g}$ such that:

$$
\begin{aligned}
& \text { if }\left|\tau_{\text {eff }}^{g}\right|<\tau_{c}^{g} \text { then } \dot{\gamma}^{g}=\dot{\gamma}_{a n}^{g}, \\
& \text { if }\left|\tau_{\text {eff }}^{g}\right| \geq \tau_{c}^{g} \text { then } \dot{\gamma}^{g}=\dot{\gamma}_{i n}^{g} .
\end{aligned}
$$

Thus, a system is considered to be active (in the sense of inelastic glide) if the condition (14) is fulfilled.

The evolution of the critical shear stresses describes the isotropic hardening. The critical shear stress of the $g^{\text {th }}$ slip system is expressed as follows:

$$
\tau_{c}^{g}=\tau_{c 0}^{g}+\frac{k}{\sqrt{d}}+\frac{1}{2} \mu b^{g} \sqrt{\sum_{h} a^{g h} \rho^{h}}+\frac{\mu b^{g}}{r_{p}} \sqrt{\frac{3 f_{v}}{2 \pi}} .
$$

Four different contributions can be highlighted in the above equation:

$-\tau_{c 0}^{g}$, the contribution of the Peïerls obstacles depending on solute atoms. 
$-k / \sqrt{d}$, the influence of the grain size that is described using the phenomenological relation of Hall-Petch (Hall, 1951; Petch, 1953) where $k$ is a material parameter and $d$ the grain size.

- $\mu b^{g} / r_{p} \sqrt{3 f_{v} / 2 \pi}$, the contribution of the non-shearable precipitates whose shape is supposed spherical.

$-1 / 2 \mu b^{g} \sqrt{\sum_{h} a^{g h} \rho^{h}}$ the hardening due to forest dislocations (Kocks, 1966). The distinction between self and latent hardening is done thanks to the matrix $a^{g h}$ (Franciosi, 1985 ) that can be defined with two coefficients $a_{1}=1$ (for coplanar systems) and $a_{2}=1.1$ (for non-coplanar systems) in the case of steel (Lipinski and Berveiller, 1989). The dislocation density evolution is described with the relation proposed by Mecking (1981) and Tabourot et al. (1997):

$$
\dot{\rho}^{g}=\frac{1}{b^{g}}\left(\frac{1}{L^{g}}-2 y_{c} \rho^{g}\right)\left|\dot{\gamma}_{i n}^{g}\right|
$$

where $y_{c}$ is the dislocation annihilation length and $L^{g}$ the free mean path of dislocations. $L^{g}$ is estimated with the following relation that takes into account the fact that the free mean path decreases when the dislocation density increases (Estrin and Mecking, 1984):

$$
\frac{1}{L^{g}}=\frac{1}{d}+\frac{\sqrt{\sum_{h} a^{g h} \rho^{h}}}{K} .
$$

where $K$ is a material parameter.

\subsection{Single crystal constitutive equation and internal variables}

Adopting the small deformation theory, the local strain $\varepsilon$ is written as the sum of an elastic, an anelastic and an inelastic strain (i.e. a Bürgers model):

$$
\varepsilon=\varepsilon^{e}+\varepsilon^{a n}+\varepsilon^{i n} .
$$

If $c$ is the local elastic moduli tensor defined from the Lamé coefficients ( $\mu$ and $\lambda$ ) with $s=c^{-1}$, the local stress field is such that:

$$
\sigma=c: \varepsilon^{e} .
$$

As the dissipative mechanisms result from dislocation displacements, the framework of crystal plasticity is chosen in order to account for the crystallographic orientation dependency. Considering that $N_{a n}$ and $N_{i n}$ are respectively the number of slip systems fulfilling conditions (13) and (14), the anelastic and inelastic strain rates can be deduced from the slip rates using the following relations:

$$
\dot{\varepsilon}^{a n}=\sum_{g}^{N_{a n}} R^{g} \dot{\gamma}_{a n}^{g} \text { and } \dot{\varepsilon}^{i n}=\sum_{g}^{N_{i n}} R^{g} \dot{\gamma}_{i n}^{g}
$$

with:

$$
R^{g}=\frac{1}{2}(\vec{m} \otimes \vec{n}+\vec{n} \otimes \vec{m}) .
$$

$\vec{m}$ and $\vec{n}$ are respectively the slip direction $(<111>)$ and the slip plane normal vectors $(\{110\}$ or $\{112\})$. The effective shear stress used to determine the anelastic and inelastic slip rates is determined from the local and kinematic stresses, $\sigma$ and $x$ :

$$
\tau_{\text {eff }}^{g}=R^{g}:(\sigma-x)=\tau^{g}-R^{g}: x
$$


where $x$ is the kinematic stress due to the inhomogeneity of the intragranular inelastic strain field. The kinematic stress tensor $x$ is calculated from the phenomenological relation proposed by Armstrong and Frederick (1966):

$$
\dot{x}=\frac{2}{3} a_{c} \dot{\varepsilon}^{i n}-c_{c} x \sqrt{\frac{2}{3} \dot{\varepsilon}^{i n}: \dot{\varepsilon}^{i n}}
$$

where $a_{c}$ and $c_{c}$ are material parameters. It is worth noticing that intragranular kinematic hardening is expected to be negligible at the range of plastic strain of interest, i.e. lower than $5 \times 10^{-4}$ (Sommer et al., 1998), namely at the range of the microyield stress. However, because some of the material parameters are identified from LCF (low cycle fatigue) stress-strain curves, intragranular hardening is required to describe the strong Bauschinger effect which is observed. Thus, in a subsequent work, to get a model integrating the main deformation and hardening mechanisms over a large range of stress amplitudes, it would be interesting to introduce more sophisticated and physically-based models such as proposed by (Mughrabi, 1983; Langlois and Berveiller, 2003; Saai et al., 2007; Sauzay, 2008). However, these physical descriptions of kinematic hardening have been developed for fcc (face-centered cubic) metals and have never been adapted to the case of bcc metals such as ferritic steels.

Finally, the local constitutive law is assimilated to a Burgers element so the local strain rate is expressed as:

$$
\dot{\varepsilon}=s: \dot{\sigma}+m^{\prime}(\sigma):\left(\sigma-c^{\prime}: \varepsilon^{a n}\right)+m(\sigma): \sigma
$$

where $m^{\prime}$ is the tensor of secant viscous compliances related to the friction when pinned dislocations oscillate and $c^{\prime}$ the tensor of elastic-type moduli related to the line tension of bended dislocations, both associated with the anelastic strain:

$$
m^{\prime}=\sum_{g}^{N_{a n}} \frac{\rho_{m}^{g} b^{g^{2}}}{f} \frac{\tau_{\text {eff }}^{g}}{\tau^{g}} R^{g} \otimes R^{g}
$$

and

$$
c^{\prime-1}=\sum_{g}^{N_{a n}} \frac{l^{g^{2}} \rho_{m}^{g}}{8 \mu} \frac{\tau_{\text {eff }}^{g}}{\tau^{g}} R^{g} \otimes R^{g} .
$$

$m$ is the tensor of secant viscous compliances associated with the inelastic strain (with $\left.b=m^{-1}\right)$ :

$$
m=\sum_{g}^{N_{\text {in }}} \frac{\dot{\gamma}_{0}}{\tau^{g}}\left(\frac{\tau_{\text {eff }}^{g}}{\mu}\right)^{2} \exp \left(\frac{-\Delta G_{0}}{k_{b} T}\left(1-\left(\frac{\left\langle\left|\tau_{\text {eff }}^{g}\right|-\tau_{c}^{g}\right\rangle}{\hat{\tau}}\right)^{p}\right)^{q}\right) \operatorname{sign}\left(\tau_{\text {eff }}^{g}\right) R^{g} \otimes R^{g}
$$

The present model aims at determining not only the mechanical response but also the dissipation. For a single crystal, the viscous power $p_{v}$ is either dissipated into heat or stored in the material. The viscous power $p_{v}$ is defined as:

$$
p_{v}=\sigma: \dot{\varepsilon}^{i n}+\left(\sigma-c^{\prime}: \varepsilon^{a n}\right): \dot{\varepsilon}^{a n}
$$

The stored power $p_{s}$ associated with isotropic (dislocations) and kinematic (dislocation arrangements) hardening is such as:

$$
p_{s}=x: \dot{y}+\sum_{g}^{N_{i n}}\left(\tau_{c}^{g}-\tau_{0}\right) \dot{q}^{g}
$$


where $y$ and $q^{g}$ are respectively the internal variables associated with the evolution of the kinematic stress tensor $x$ and the critical shear stress $\tau_{c}^{g}$. Following the proposition of Lemaitre et al. (2009), the evolution rate of the internal variable $y$ is given by:

$$
\dot{y}=\dot{\varepsilon}^{i n}-\frac{3 c_{c}}{2 a_{c}} x \sqrt{\frac{2}{3} \dot{\varepsilon}^{i n}: \dot{\varepsilon}^{i n}}
$$

such that:

$$
x=\frac{2}{3} a_{c} y
$$

Following the work of Sarma et al. (1998), the stored power associated with isotropic hardening can be associated to the dislocation density evolution rate so:

$$
\left(\tau_{c}^{g}-\tau_{0}\right) \dot{q}^{g}=\frac{1}{2} \mu b^{g^{2}} \dot{\rho}^{g} .
$$

From the above equation, the expression of $\dot{q}^{g}$ is deduced:

$$
\dot{q}^{g}=\frac{\dot{\rho}^{g}}{b^{g} \sqrt{\sum_{h} a^{g h} \rho^{h}}} .
$$

Finally, the expression of the average dissipation per cycle $d_{1}$ is obtained:

$$
d_{1}=\frac{1}{T_{s}} \int_{t}^{t+T_{s}}\left(p_{v}-p_{s}\right) d t
$$

\section{Scale transition model}

In the Results section, some predictions from the proposed modeling will be compared with experimental results obtained for different ferritic steel grades. To estimate the overall mechanical and dissipative behavior of ferritic polycrystals of interest, we consider a representative volume element (RVE) including a very large number of heterogeneities (grains). The overall behavior is deduced using a homogenization scheme developed for elastic-anelastic-inelastic heterogeneous materials (Mareau et al., 2009a). Such a behavior involves strong space-time couplings and time-dependent mechanical interactions because of the presence of different time derivation orders in the local constitutive equation (24). Although hereditary approaches are well suited to estimate the interactions in heterogeneous materials with such a constitutive law (Rougier et al., 1994; Masson and Zaoui, 1999), they are very time consuming because of the inverse Laplace-Carson transformation that needs to be performed. Therefore, we prefer to use the scale transition model of Mareau et al. (2009a). This model is based on an incremental approach and internal variables which contain the mechanical history of the material. As a result, the mechanical history is not required to be stored all test long which is less time and memory consuming.

The average stress rate is determined in each grain using the following interaction law that was established with translated field techniques:

$$
\dot{\sigma}=B^{C}: \dot{\Sigma}+c: A^{C}:\left(S^{E C}-I\right):\left(\dot{\varepsilon}^{a n}+\dot{\varepsilon}^{i n}-\dot{e}^{v}\right)
$$

where $I$ is the fourth-rank identity tensor, $B^{C}$ and $S^{E C}$ are respectively the stress concentration tensor and the Eshelby tensor (Eshelby, 1957) associated with the equivalent homogeneous medium with purely elastic properties determined using the self-consistent 
approximation (Kröner, 1958) and $\dot{e}^{v}$ is a translated field that depends on the macroscopic inelastic strain rate field $\dot{E}^{i n}$ :

$$
\dot{e}^{v}=A^{B}: \dot{E}^{i n}+A^{B}: \dot{e}^{a n}+A^{B}: S^{E B}:\left(M: b: \dot{\varepsilon}^{a n}-\dot{e}^{a n}\right) .
$$

$A^{B}, M$ and $S^{E B}$ are respectively the strain concentration tensor, the compliances tensor and the Eshelby tensor associated with the equivalent homogeneous medium with purely inelastic properties determined using the self-consistent approximation and $\dot{e}^{a n}$ is a translated field that depends on the macroscopic anelastic strain rate field $\dot{E}^{a n}$ :

$$
\dot{e}^{a n}=A^{B^{\prime}}: \dot{E}^{a n}-A^{B^{\prime}}: S^{E B^{\prime}}: M^{\prime}:\left(c^{\prime}: e^{a n}-c^{\prime}: A^{C^{\prime}}: E^{a n}\right) .
$$

$A^{B^{\prime}}, M^{\prime}$ and $S^{E B^{\prime}}$ are respectively the strain concentration tensor, the compliances tensor and the Eshelby tensor associated with the equivalent homogeneous medium with purely viscous anelastic properties determined using the self-consistent approximation and $A^{C^{\prime}}$ is the strain concentration tensor associated with the equivalent homogeneous medium with purely non-viscous anelastic properties determined using the self-consistent approximation.

The present scale transition model is based on the introduction of the translated fields $\dot{e}^{a n}$ and $\dot{e}^{v}$ so that the real fields are written as the sum of a reference strain rate field and some fluctuations $\delta \dot{\varepsilon}^{a n}$ and $\delta \dot{\varepsilon}^{v}$ :

$$
\begin{gathered}
\dot{\varepsilon}^{a n}=\dot{e}^{a n}+\delta \dot{\varepsilon}^{a n} \\
\dot{\varepsilon}^{v}=\dot{\varepsilon}^{a n}+\dot{\varepsilon}^{i n}=\dot{e}^{v}+\delta \dot{\varepsilon}^{v}
\end{gathered}
$$

The translated fields are chosen as the solutions of the purely anelastic heterogeneous problem and the purely viscous (i.e. anelastic and inelastic) heterogeneous problem, respectively. The presence of fluctuations enables to obtain an accurate estimation of the interactions between grains when the self-consistent approximation is performed. Further details are given in Mareau et al. (2009a).

The (volume and time) average dissipation per cycle $\tilde{d}_{1}$ is simply determined using the following expression:

$$
\tilde{d}_{1}=\frac{1}{V} \int_{V} d_{1} d V
$$

where $V$ is the volume of the RVE.

\section{Results}

\subsection{Assessment of the modeling}

We simulated cyclic loading tests with the micro-macro model using triangular waveform of tension-compression load with the frequency of $30 \mathrm{~Hz}$ and for different stress amplitudes. We compared the predicted stress-strain curves to experimental results obtained for low alloyed ferritic steel grades hardened with non-shearable precipitates. The first grade is a HSLA (High-Strength Low-Alloy) 320 steel. Its conventional yield stress is $369 \mathrm{MPa}$ and the volume fraction of precipitates is $31 \mathrm{ppm}$. The second grade is a HSLA 420 steel. Its conventional yield stress is $505 \mathrm{MPa}$ and the volume fraction of precipitates is $345 \mathrm{ppm}$. Note that experimental tests were conducted using sinusoidal 
rather than triangular waveform but with the same tension-compression load and frequency as for simulations. Experiments were achieved on specimens with a low surface roughness (i.e. $R_{a}<0.050 \mu \mathrm{m}$ ). This geometric condition ensures that the free surface effect on dislocation activities is not predominant with regard to bulk phenomena so that the concept of a representative volume element is valid and we can access to the impact of microstructural parameters on the mechanical and dissipative responses (Mareau et al., 2009b). Since the measured texture of the studied steel grade is nearly random, the RVE consists of 2000 discrete orientations representing a random cubic texture.

Parameters of the model are listed in Table 1. The size and the volume fraction of precipitates and the mean grain size were obtained from microstructure observations. Physical parameters such as the magnitude of the Burgers vector and the elastic Lamé coefficients are well-known and prescribed from literature data. The other parameters were identified to match the LCF strain-stress curves that were experimentally obtained (see Figure 5). This choice is motivated by the fact that the stress-strain hysteresis loops at the microyield stress range are too thin to properly identify material parameters associated with inelastic slip rate and critical shear stress equations (see equations (12) and (15)). However, it required to introduce intragranular kinematic hardening. Indeed and as mentioned in section 2.2, intergranular kinematic hardening inherently accounted by the interaction law (equation (35)) is not sufficient to capture the strong Bauschinger effect observed in the LCF stress-strain curves. It is worth noticing that this intragranular kinematic hardening is second order for dissipation estimation at the microyield stress range. Indeed, predicted $x$ values are much lower than the local stresses $(x$ does not exceed $0.05 \sigma)$. Note that $\dot{\gamma}_{0}, p, q, \Delta G_{0}$ and $y_{c}$ were bounded to agree with the values reported in literature for bcc ferritic steels (Essmann and Mughrabi, 1979; Frost and Ashby, 1982; Harder, 1999; Paquin et al., 2001; Berbenni et al., 2004). The comparison between experimental and predicted stress-strain curves shows that isotropic hardening is slightly overestimated for a $0.6 \%$ or a $0.8 \%$ maximal strain while it is underestimated for a $0.4 \%$ maximal strain. The description of isotropic hardening could be improved either by changing the constitutive law or with a better adjustment of hardening parameters. However, the present model in conjunction with the current identification procedure leads to a good quantitative agreement between experimental and predicted results demonstrating it is able to capture the cyclic stress-strain curves in a stress range around the macroscopic yield stress.

\begin{tabular}{|c|c|c|c|c|}
\hline$\mu(\mathrm{MPa})$ & $\lambda(\mathrm{MPa})$ & $b(\mathrm{~m})$ & $1 / f(\mathrm{~Pa} . \mathrm{s})^{-1}$ & $\rho\left(\mathrm{m}^{-2}\right)$ \\
70000 & 105000 & $2.5 \times 10^{-10}$ & 8000 & $10^{10}$ \\
\hline \hline$\dot{\gamma}_{0}\left(\mathrm{~s}^{-1}\right)$ & $p$ & $q$ & $\Delta G_{0} / k_{b}(\mathrm{~K})$ & $T(\mathrm{~K})$ \\
9100 & 0.05 & 1 & 15000 & 293 \\
\hline \hline$\hat{\tau}(\mathrm{MPa})$ & $\tau_{c 0}+k / \sqrt{d}(\mathrm{MPa})$ & $d(\mu \mathrm{m})$ & $f_{v}(\mathrm{ppm})$ & $r_{p}(\mathrm{~m})$ \\
50 & 60 & 13 & 31 & $5 \times 10^{-9}$ \\
\hline \hline$\alpha$ & $y_{c}(\mathrm{~m})$ & $K$ & $a_{c}(\mathrm{MPa})$ & $c_{c}$ \\
0.5 & $4 \times 10^{-8}$ & 80 & $1.8 \times 10^{5}$ & 2200 \\
\hline
\end{tabular}

Table 1

Identified parameters for a HSLA 320 steel grade. 


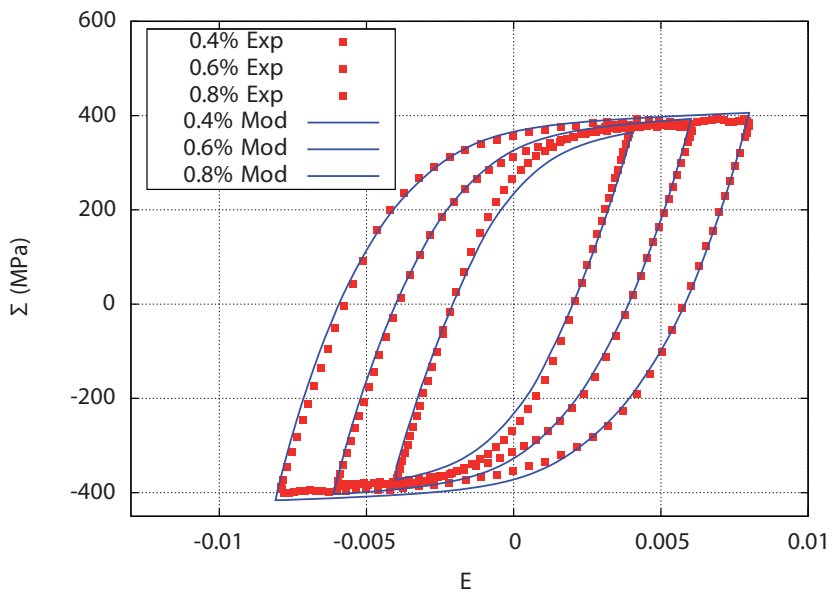

Fig. 5. Experimental (Exp) and predicted (Mod) stress-strain curves in LCF for a HSLA 320 steel grade different maximal strains in order to fit the parameters.

In order to plot the predicted self-heating curves and to compare them to experimental results, we simulated a cyclic loading $\left(R_{s}=-1, f_{s}=30 \mathrm{~Hz}\right)$ up to 20 cycles (which is the necessary number of cycles to get stabilized hysteresis loops) for stress amplitude ranging from 50 to $230 \mathrm{MPa}$. Note that, in these simulations, the stress range is lower than the stress range we used above for identification. Curves associated with Figure 6 show a good agreement between experimental and predicted self-heating curves in both regimes. The strong increase of dissipation induced by plastic slip is well captured by the model. Better results could be obtained if a more accurate value of the friction coefficient was known. However, because of the very low anelastic strains, its identification from LCF stress-strain curves is not accurate.

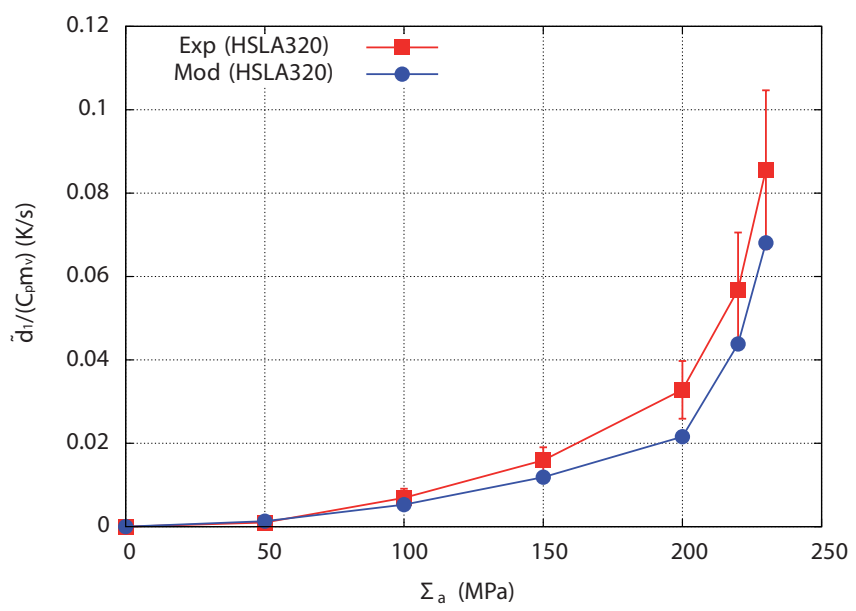

Fig. 6. Comparison between experimental (Exp) and predicted (Mod) self-heating curves, namely average dissipation per cycle versus stress amplitude, for a HSLA 320 steel grade.

Next, the self-heating curve for an another HSLA steel grade having much more pre- 
cipitates were simulated using the same model parameters except the volume fraction of precipitates which was changed to be consistent with experimental values (i.e. $f_{v}=345$ $\mathrm{ppm}$ ). A qualitative agreement is found between experimental and calculated data (Fig. 7). The dissipation is reduced in the first regime, with regards to results for HSLA with 31 ppm. In addition, the first regime is stretched to larger macroscopic stresses. Increasing the volume fraction of the precipitates results in shortening the pinned dislocation lines and as a consequence reduces the dissipation. Besides, the critical shear stress required to multiply dislocations increases with the volume fraction of precipitates. This is why the second regime of dissipation occurs for higher stress amplitudes. However some discrepancies appear between experimental and simulated results: the model underestimates the dissipation in the first regime and overestimates the dissipation in the second regime. In other words, the predicted transition from the anelastic regime to the inelastic regime is too pronounced in comparison with the experimental self-heating curve. The explanation for the poor description of the transition is twofold. On the one hand, for the sake of simplicity, we consider the mean behavior of dislocations of a given slip system although the dislocations of a given slip system can have different (anelastic or inelastic) behaviors. This hypothesis is consistent with the crystal plasticity framework but provides a dramatic transition between the two regimes (the dissipation jump is around $0.01 \mathrm{~K} / \mathrm{s}$ at the slip system scale). On the other hand, because of the self-consistent formulation, only the average quantities (i.e. stress, strain, dissipation) are considered to describe the behavior of a given grain while the plastic activity (so the dissipation) might be localized in some regions where deformation conflicts are important (e.g. grain boundaries). The inelastic regions can progressively grow with an increasing stress resulting in a smooth anelasticinelastic transition. However, the possibility to have some regions of a given grain with a predominant inelastic behavior and some others with a predominant anelastic behavior is not considered in the present modeling.

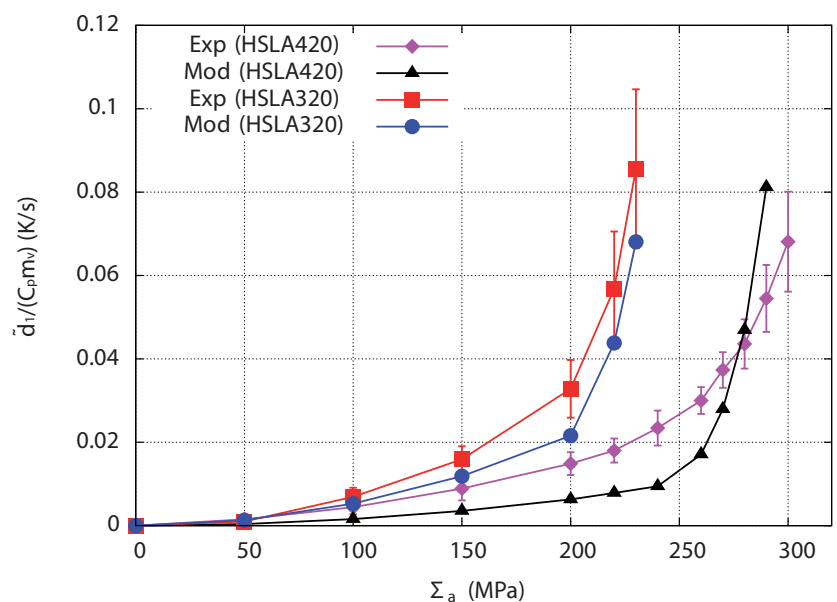

Fig. 7. Comparison between experimental (Exp) and predicted (Model) self-heating curves, namely average dissipation per cycle versus stress amplitude, for two HSLA steel grades having different volume fractions of precipitates (i.e. 31 and $345 \mathrm{ppm}$ ) 


\subsection{Effect of microstructure}

In order to optimize the microstructure of steels, it is necessary to understand how it interacts with the dissipative mechanisms. The model was used to see how the mean grain size, the grain size dispersion and the crystallographic orientation affect the selfheating curves. To have consistent parameters in order to perform realistic calculations, the parameters given in table 2 were used for the simulations presented in this section unless otherwise specified. The parameters were identified for a $100 \%$ ferritic steel (IF 280) from LCF stress-strain curves.

\begin{tabular}{|c|c|c|c|c|}
\hline$\mu(\mathrm{MPa})$ & $\lambda(\mathrm{MPa})$ & $b(\mathrm{~m})$ & $1 / f(\mathrm{~Pa} . \mathrm{s})^{-1}$ & $\rho\left(\mathrm{m}^{-2}\right)$ \\
75000 & 112500 & $2.5 \times 10^{-10}$ & 8000 & $10^{10}$ \\
\hline \hline$\dot{\gamma}_{0}\left(\mathrm{~s}^{-1}\right)$ & $p$ & $q$ & $\Delta G_{0} / k_{b}(\mathrm{~K})$ & $T(\mathrm{~K})$ \\
7400 & 0.05 & 1 & 15000 & 293 \\
\hline \hline$\hat{\tau}(\mathrm{MPa})$ & $\tau_{c 0}(\mathrm{MPa})$ & $d(\mu \mathrm{m})$ & $f_{v}(\mathrm{ppm})$ & $k\left(\mathrm{MPa} . \mu \mathrm{m}^{1 / 2}\right)$ \\
50 & 50 & 12 & 0 & 70 \\
\hline \hline$\alpha$ & $y_{c}(\mathrm{~m})$ & $K$ & $a_{c}(\mathrm{MPa})$ & $c_{c}$ \\
0.5 & $4.10^{-8}$ & 100 & $3.10^{5}$ & 3500 \\
\hline
\end{tabular}

Table 2

Identified parameters for an IF 280 steel grade.

\subsubsection{Mean grain size}

The mean grain size is known to strongly influence mechanical properties of metallic materials - the fatigue limit in particular (Klesnil and Lukas, 1992). Assuming homogeneous grain size in the RVE, we calculated the self-heating curves for three different mean grain sizes (see figure 8). The results show that the average dissipation per cycle is not grain size sensitive in the anelastic regime but significantly decreases with decreasing grain size consistently with experimental results (Doudard, 2004). These results are explained by the Hall-Petch effect: when grains are smaller, the critical shear stress is higher. This effect is modeled at the slip scale in the present modeling by equation (15).

\subsubsection{Grain size dispersion}

In metallic alloys such as steel, the dispersion of grain size is partly responsible for the heterogeneity of the material. Indeed, Kurzydlowski (1990) and Berbenni et al. (2007) showed that the yield strength depends on both the mean value and the standard deviation of the grain size. In polycrystals, the grain size generally obeys a log-normal law (Rhines and Patterson, 1982; Ter Heege et al., 2004). Assuming a log-normal grain size distribution fully independent on the crystallographic orientation, three unimodal distributions with the same mean grain size $(\bar{d}=10 \mu \mathrm{m})$ but different dispersions were generated and then discretized (see figure 9) to build three different RVEs with a random texture. In the case of a log-normal distribution, the probability density function $P$ associated with the variable $d$ is given by: 


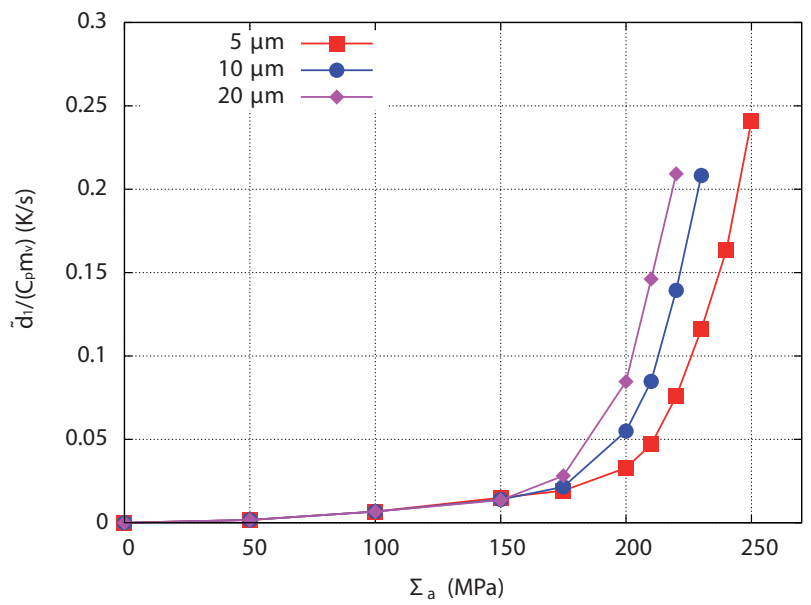

Fig. 8. Self-heating curves, namely average dissipation per cycle versus stress amplitude, calculated with the model for different mean grain sizes: $\bar{d}=5, \bar{d}=10$ and $\bar{d}=20 \mu \mathrm{m}$.

$$
P(d)=\frac{1}{\mu_{d} s_{d} \sqrt{2 \pi}} \exp \left(-\frac{\left(\log d-\mu_{d}\right)^{2}}{2 s_{d}^{2}}\right)
$$
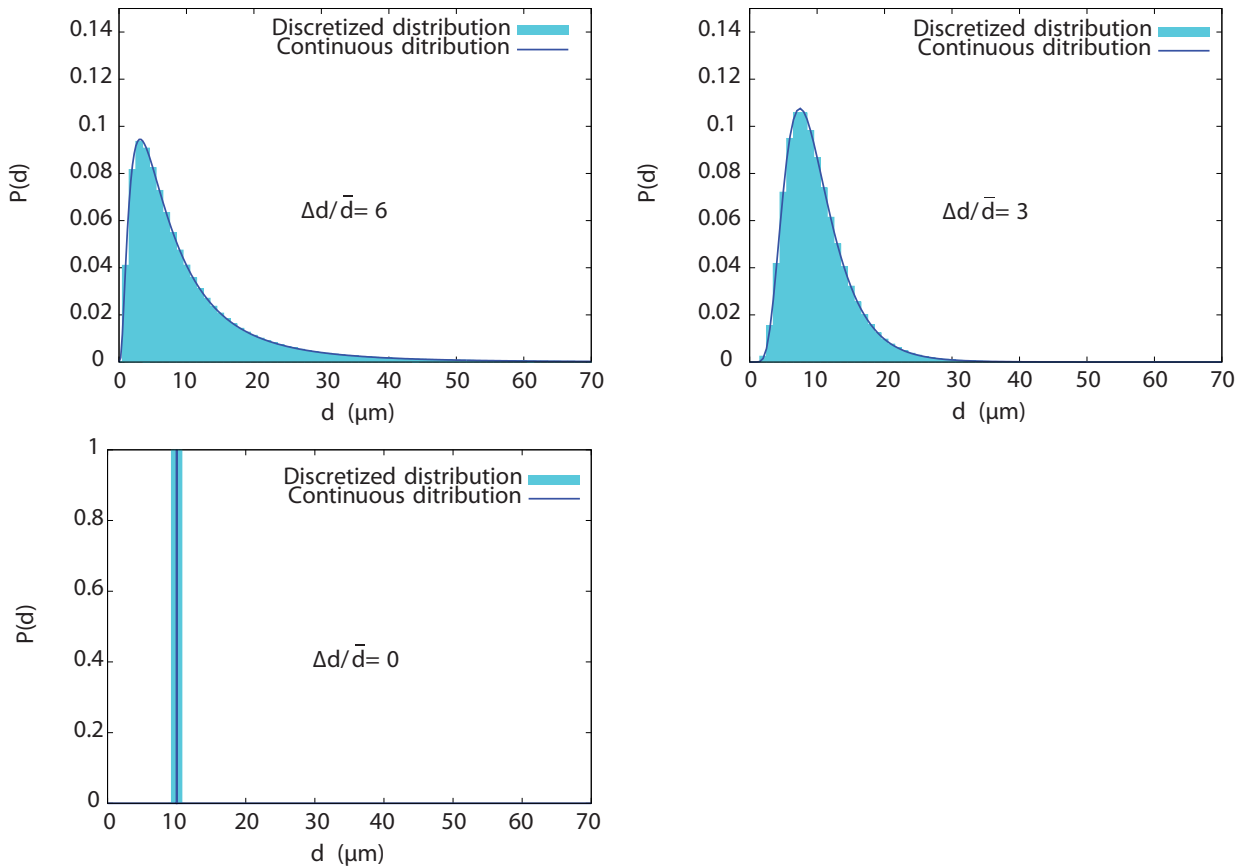

Fig. 9. Grain size distributions used to build three different fictive polycrystals: $\Delta d / \bar{d}=6, \Delta d / \bar{d}=3$ and $\Delta d / \bar{d}=0$.

where $\mu_{d}$ and $s_{d}$ are respectively the mean and standard deviation of the grain size natural logarithm (i.e. the logarithm of the grain size is normally distributed). The self- 
heating curves associated with the different grain size dispersions were calculated using the model and are plotted on figure 10. We observe that increasing the grain size dispersion increases the average dissipation per cycle in the inelastic regime. Such an influence is due to larger inelastic strains occurring in the coarsest grains because of their low local yield stress and so that are highly dissipative. It should be noticed that the impact of the grain size dispersion is comparable to the influence of the mean grain size.

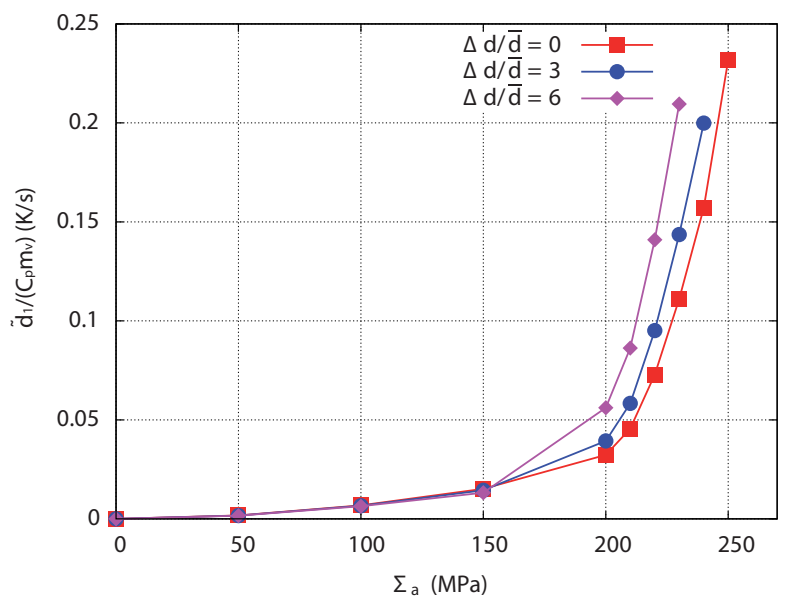

Fig. 10. Heating curves, namely average dissipation per cycle versus stress amplitude, calculated with the model for different grain size distributions: $\Delta d / \bar{d}=6, \Delta d / \bar{d}=3$ and $\Delta d / \bar{d}=0$.

The range of the dissipation field $\left(\Delta \tilde{d}_{1}=\operatorname{Max}\left(d_{1}\right)-\operatorname{Min}\left(d_{1}\right)\right)$ was calculated as a function of the stress amplitude for the different grain size distributions (see figure 11). The dissipation field scatter is obviously higher when the grain size dispersion increases. In the inelastic regime, the range of dissipation is very large around the mean value of dissipation which emphasizes the fact that the dissipation tends to be concentrated in the biggest grains. Considering the place of highest dissipation as a likely microcrack initiation location, these results highlight the fact that not only the mean grain size but also the dispersion have to be controlled to improve fatigue properties.

\subsubsection{Crystallographic orientation}

Crystallographic orientation is another heterogeneity source for the mechanical fields. To estimate the impact of the crystallographic orientation heterogeneity, the local dissipation field was studied with the present model. Using the RVE with an uniform grain size $(\Delta d / \bar{d}=0)$ and a random initial texture, the average dissipation per cycle was calculated for each orientation for a given stress amplitude $\left(\Sigma_{a}=250 \mathrm{MPa}\right)$. The local average dissipation per cycle $d_{1}$ is plotted as a function of the maximal Schmid factor (i.e. $\max \left(\left|R_{i i}^{g}\right|\right)_{g=1,24}$ where $i$ defines the loading direction) on figure 12. The results emphasize the strong dispersion of the dissipation sources due to the various grain crystallographic orientations. In general, the dissipation is found the highest for grains the most favorably oriented to slip for uniaxial tension-compression according to the Schmid law. Some grains exhibit the highest dissipation degree though their maximal Schmid factor is smaller than 0.5 (but higher than 0.46) because multiple systems are active in those grains. Indeed, the maximal Schmid factor indicates the orientation of a single slip 


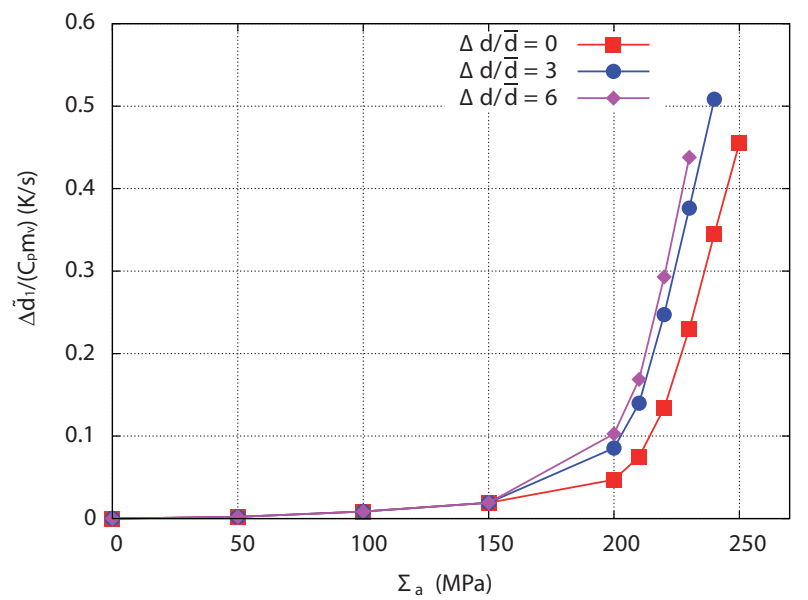

Fig. 11. Range of the dissipation field calculated with the model for different grain size distributions $(\Delta d / \bar{d}=6, \Delta d / \bar{d}=3$ and $\Delta d / \bar{d}=0)$ as a function of the stress amplitude.

system but does not preclude multi slips. In addition, the average dissipation over the polycrystalline volume was found equal to $0.25 \mathrm{~K} / \mathrm{s}$. We calculated that the most dissipative grains (i.e. with $d_{1}>0.40 \mathrm{~K} / \mathrm{s}$ ) provide about $25 \%$ of the total dissipation though their total volume fraction is about $10 \%$. As for the grain size distribution, this result suggests that, if one considers the most dissipative grains as some potential microcrack initiation locations, the dispersion of the dissipation field associated with the distribution of crystallographic orientations must be accounted for.

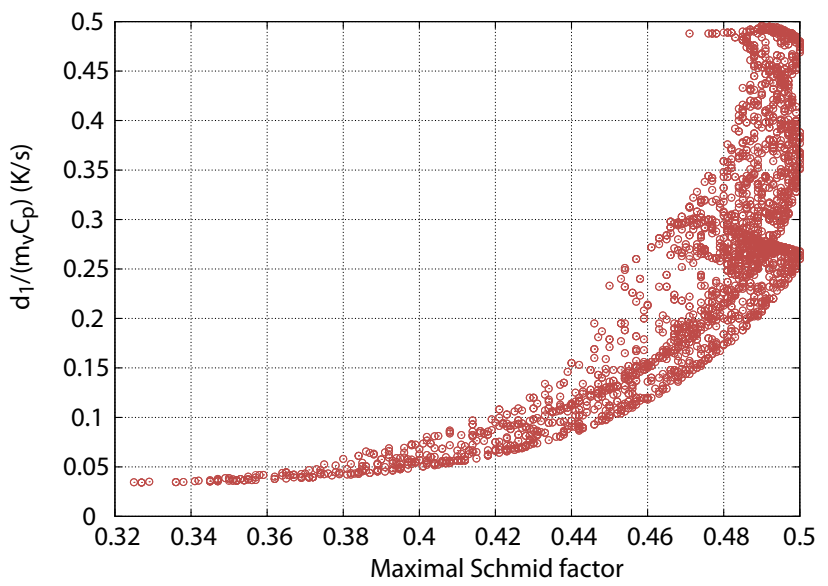

Fig. 12. Calculated average dissipation per cycle per grain as a function of the maximal Schmid factor.

\section{Concluding remarks}

A micromechanical model was proposed in order to describe the dissipative mechanisms in steels under cyclic loading. Two different mechanisms were assumed to be responsible 
for heat dissipation: the oscillation of pinned dislocations defined as an anelastic mechanism and the viscoplastic slip of dislocations considered as an inelastic mechanism. An original relation was proposed to model the anelastic slip of dislocations at the scale of the slip system. The single crystal constitutive law was built by using the framework of crystal plasticity. The transition to the polycrystalline scale was performed thanks to a mean field approach that takes into account the couplings between elastic, anelastic and inelastic mechanisms and the induced complex mechanical interactions between heterogeneities.

Here we have compared the micromechanical predictions for self-heating curves with experimental findings for HSLA steels with different volume fractions of precipitates. The present model successfully captures the dissipation change as a function of the stress amplitude, namely the first regime of low dissipation associated with anelastic mechanisms and the second regime of much higher dissipation induced by inelastic mechanisms. It describes qualitatively the impact of precipitates on dissipation observed in the experiments: (i) the presence of precipitates reduces the pinned dislocation length and consequently reduces the displacement amplitude of the oscillations and so the dissipation and (ii) the precipitates act as obstacles to dislocation glide and so higher stresses are required to have significant plastic strain and dissipation.

The influence of mean grain size, grain size distribution and grain orientation distribution on the self-heating curves was analyzed. The dissipation is not grain size sensitive in the anelastic regime but decreases with decreasing grain size in the second regime, as expected from experimental findings. Predicted results demonstrate the key role of dispersion of grain size and orientation. Both type of dispersion induce very large dispersion of dissipation. Large and well-oriented grains for plasticity are about 30 times more dissipative than other grains and so are preferential sites for microcrack initiation. These results reveal that controlling mean grain size but also grain size distribution is of a great interest for optimizing microstructures for fatigue properties. More generally, particular attention has to be paid to the microstructure parameters contributing to the heterogeneity of the mechanical fields.

\section{References}

Armstrong, P.J., Frederick C.O., 1966. A mathematical representation of the multiaxial Bauschinger effect. Technical Report RD/B/N 731, Central Electricity Generating Board.

Berbenni, S., Favier, V., Lemoine, X., Berveiller, M., 2004. Micromechanical modeling of the elastic-viscoplastic behavior of polycrystalline steels having different microstructures. Materials Science and Engineering A 372 (1-2), 128-136.

Berbenni, S., Favier, V., Berveiller, M., 2007. Impact of the grain size distribution on the yield stress of heterogeneous materials. Int. J. Plast. 23 (1), 114-142.

Berthel, B., Wattrisse, B., Chrysochoos, A., Galtier, A., 2007. Thermographic Analysis of Fatigue Dissipation Properties of Steel Sheets. Strain 43 (3), 273-279.

Boulanger, T., Chrysochoos, A., Mabru, C., Galtier, A., 2004. Calorimetric analysis of dissipative and thermoelastic effects associated with the fatigue behavior of steels. International Journal of Fatigue 26, 221-229.

Boulanger, T., 2004. Analyse par thermographie infrarouge des sources de chaleur in- 
duites par la fatigue des aciers. PhD Thesis, Université Montpellier II.

Brown, N., Ekvall, R.A., 1962. Temperature dependence of the yield points in iron. Acta Metall. 10, 1101-1107.

Campbell, J.D., Ferguson, W.G., 1970. The temperature and strain-rate dependence of the shear strength of mild steel. Phil. Mag. 21 (169), 63-82.

Chrysochoos, A., Berthel, B., Latourte, F., Pagano, S., Wattrisse, B., Weber, B., 2008. Local Energy Approach to Steel Fatigue. Strain 44 (4), 327-334.

Connesson, N., Maquin, F., Pierron, F., 2010. Experimental energy balance during the first cycles of cyclically loaded specimens under the conventional yield stress. Exp. Mech. 51 (1), 23-44.

Deschamps, A., Brechet Y., 1998. Influence of predeformation and ageing of an Al-ZnMg alloy II. Modeling of precipitation kinetics and yield stress. Acta Materialia 47 (1), 293-305.

Doudard, C., 2004. Détermination rapide des propriétés en fatigue à grand nombre de cycles à partir d'essais d'échauffement. PhD Thesis, ENS Cachan.

Eshelby, J.D., 1957. The determination of the elastic field of an ellipsoidal inclusion, and related problems. Proc. Roy. Soc. A241, 376-396.

Essmann, U., Mughrabi, H.,1979. Annihilation of dislocation during tensile and cyclic deformation and limits of dislocation densities. Phil. Mag. A 40, 731-756.

Estrin, Y., Mecking, H., 1984. A unified phenomenological description of work hardening and creep based on one-parameter models. Acta Metall. 32, 57-70.

Franciosi, P., 1985. The concepts of latent hardening and strain hardening in metallic single crystals. Acta Metall. 33 (9), 1601-1612

Friedel, J., 1964. Dislocations, Pergamon Press, Oxford.

Frost, H.J., Ashby, M.F., 1982. Deformation-Mechanism-Maps, the plasticity and creep of metals and ceramics. Pergamon Press, Oxford, UK.

Galtier, A., Bouaziz, O., Lambert, A., 2002. Influence de la microstructure des aciers sur leurs propriétés mécaniques. Méc. Ind. 8, 457-462.

Gomaa, A., 1980. Corrélation entre la dissipation thermique d'une éprouvette en torsion alternée et sa limite d'endurance en fatigue. PhD Thesis, Université Bordeaux 1.

Granato, A., Lücke, K., 1956. Theory of mechanical damping due to dislocations. J. Appl. Phys. 27 (6), 583-592.

Hall, E.O., 1951. The deformation and ageing of mild steel. Proc. Phys. Soc. London B64, 747-753.

Harder, J., 1999. A crystallographic model for the study of local deformation processes in polycrystals. Int. J. Plasticity 15, 605-624.

Johnston, W.G., Gilman J.J., 1959. Dislocation Velocities, Dislocation Densities, and Plastic Flow in Lithium Fluoride Crystals. J. Appl. Phys. 30, 129.

Klesnil, M., Lukas, P., 1992. Fatigue of metallic materials, Elsevier.

Kocks, U.F., 1966. A statistical theory of flow stress and work-hardening. Phil. Mag. 13, 541-566.

Koehler, J.S., 1952. Imperfections in Nearly Perfect Crystals, in: Shockley, W., Hollomon, J.H., Maurer, R., Seitz, F.,(Eds.), Proceedings of a Symposium held at Pocono Manor, Penna., U. S. A., Wiley and Sons, New York, pp. 197.

Kröner, E., 1958. Berechnung der elastischen Konstanten des Vielkristalls aus den Konstanten des Einskristalls. Z. Phys., 151.

Kurzydlowski, K.J., 1990. A model for the flow stress dependence on the distribution of 
grain size in polycrystals. Scr. Metall. Mater. 24 (5), 879-884.

Langlois, L., Berveiller, M., 2003. Overall softening and anisotropy related with the formation and evolution of dislocation cell structures. Int. J. Plast. 19, 599624.

La Rosa, G., Risitano, A., 2000. Thermographic methodology for rapid determination of the fatigue limit of materials and mechanical components. International Journal of Fatigue 22 (1), 65-73.

Lemaitre, J., Chaboche, J.L., Benallal, A., Desmorat, R., 2009. Mécanique des matériaux solides, Dunod, Paris.

Lipinski, P., Berveiller, M., 1989. Elastoplasticity of micro inhomogeneous metals at large strains. Int. J. Plast. 5 (2), 149-172.

Louche, H., Chrysochoos, A., 2001. Thermal and dissipative effects accompanying Luders band propagation. Materials Science and Engineering A 307 (1), 15-22.

Louchet, F., Kubin, L.P., Vesely, D., 1979. In situ deformation of B.C.C. crystals at low temperatures in a high-voltage electron microscope Dislocation mechanisms and strain-rate equations. Phil. Mag. A 39 (4), 433-454.

Luong, M.P., 1998. Fatigue limit evaluation of metals using an infrared thermographic technique. Mechanics of Materials 28 (1-4), 155-163.

Maquin, F., Pierron, F., 2009. Heat dissipation measurements in low stress cyclic loading of metallic materials: From internal friction to micro-plasticity. Mechanics of Materials 41, 928-942.

Mareau, C., Favier, V., Berveiller, M., 2009a. Micromechanical modeling coupling timeindependent and time-dependent behaviors for heterogeneous materials. Int. J. of Solids Struct. 46 (2), 223-237.

Mareau, C., Favier, V., Weber, B., Galtier, A., 2009b. Influence of the free surface and the mean stress on the dissipation in steels under cyclic loading. International Journal of Fatigue 31(8-9), 1407-1412.

Masson, R., Zaoui, A., 1999. Self-consistent estimates for the rate-dependent elastoplastic behaviour of polycrystalline materials. J. Mech. Phys. Solids 47 (7), 1543-1568.

Mecking, H., 1981. Strain hardening and dynamic recovery, in: Ashby, M.F., Bullough R., Hartley C.S., Hirth J.P. (Eds), Dislocation Modelling of Physical Systems, Pergamon Press, NY, pp. 197.

Mollica, F., Rajagopal, K.R., Srinivasa, A.R., 2001. The inelastic behavior of metals subject to loading reversals. International Journal of Plasticity 17, 1119-1146.

Morabito, A.E., Dattoma, V., Galietti, U., 2002. Energy-analysis of fatigue damage by thermographic technique, in: Maldague, X.P., Rozlosnick, A.E. (Eds.), Proceedings of the 24th Thermosense conference, SPIE, pp. 460.

Mughrabi, H., 1983. Dislocation wall and cell structures and long-range internal stresses in deformed metal crystals. Acta Metall. 31 (9), 1367-1379.

Munier, R., Doudard, C., Calloch, S., Weber, B., 2010. Towards a faster determination of high cycle fatigue properties taking into account the influence of a plastic pre-strain from selfheating measurements. Procedia Engineering 2(1), 1741-1750.

Nabarro, F.R.N., 1947. Dislocations in a simple cubic lattice. Proc. Phys. Soc. Lond. 59, 256-272.

Naamane, S., Monnet, G., Devincre, B., 2010. Low temperature deformation in iron studied with dislocation dynamics simulations. Int. J. of Plast. 26, 84-92.

Paquin, A., Berbenni, S., Favier, V., Lemoine, X., Berveiller, M., 2001. Micromechanical modeling of the elastic-viscoplastic behavior of polycrystalline steels. International 
Journal of Plasticity 17 (9), 1267-1302.

Peierls, R., 1940. The size of a dislocation. Proc. Phys. Soc. Lond. 52, 34-37.

Petch, N.J., 1953. The cleavage strength of polycrystals. J. Iron Steel Inst. 174, 25-28.

Poncelet, M., Doudard, C., Calloch, S., Weber, B., Hild, F., 2010. Probabilistic multiscale models and measurements of self-heating under multiaxial high cycle fatigue. Journal of the Mechanics and Physics of Solids 58(4), 578-593.

Rhines, F.N., Patterson, B.P., 1982. Effect of the degree of prior cold work on the grain volume distribution and the rate of grain growth of recrystallized aluminium. Metall. Trans. A 13 (6), 985-993.

Rougier, Y., Stolz, C., Zaoui, A., 1994. Self-consistent modeling of elastic-viscoplastic polycrystals. C. R. Acad. Sci. Paris 318, 145-151.

Saai, A., Tabourot, L., Déprés, C., Louche, H., 2007. A fundamental model of aluminum single crystal behavior with physical description of kinematic work hardening. Materials Science Forum 550, 577-582.

Sarma, G.B., Radhakrishnan, B., Zacharia, T., 1998. Finite element simulations of cold deformation at the mesoscale. Comput. Mater. Sci. 12, 105-123.

Sauzay, M., 2008. Analytical modelling of intragranular backstresses due to deformation induced dislocation microstructures, Int. J. Plast. 24, 727-745.

Seeger, A., 2004. Progress and problems in the understanding of the dislocation relaxation processes in metals. Materials Science and Engineering A 370, 5066.

Snoek, J.L., 1941. Effect of small quantities of carbon and nitrogen on the elastic and plastic properties of iron. Physica VIII (7), 711-733.

Solomon, H.D., McMahon, C.J., 1966. Exhaustion hardening of iron, in: Work hardening - Metallurgical Society Conference, vol. 46. Gordon and Beach, New York, pp. 311-332.

Sommer, C., Mughrabi, H., Lochner, D., 1998. Influence of temperature and carbon content on the cyclic deformation and fatigue behaviour of $\alpha$-iron. Part 1. Cyclic deformation and stress-behaviour. Acta Mater. 46, 1527-1536.

Tabourot, L., Fivel M., Rauch E., 1997. Gereralized constitutive laws for FCC single crystal, Materials Science and Engineering A 234-236, 639-642.

Tang, M., Kubin, L.P., Canova, G.R., 1998. Dislocation mobility and the mechanical response of BCC single crystals: a mesoscopic approach. Acta Mater. 46 (9), 32213235 .

Ter Heege, J.H., De Bresser, J.H.P., Spiers, C.J., 2004. Composite flow laws for crystalline materials with lognormally distributed grain size: theory and application to olivine. J. Struct. Geol. 26, 1693-1705. 